University of Wollongong

Research Online

Faculty of Social Sciences - Papers (Archive) Faculty of Arts, Social Sciences \& Humanities

2019

Investigating the mediators and moderators of child body mass index change in the Time2bHealthy childhood obesity prevention program for parents of preschool-aged children

Megan L. Hammersley

University of Wollongong,mhammers@uow.edu.au

Anthony D. Okely

University of Wollongong, tokely@uow.edu.au

Marijka Batterham

University of Wollongong, marijka@uow.edu.au

Rachel A. Jones

University of Wollongong, rachelj@uow.edu.au

Follow this and additional works at: https://ro.uow.edu.au/sspapers

Part of the Education Commons, and the Social and Behavioral Sciences Commons

Research Online is the open access institutional repository for the University of Wollongong. For further information contact the UOW Library: research-pubs@uow.edu.au 


\title{
Investigating the mediators and moderators of child body mass index change in the Time2bHealthy childhood obesity prevention program for parents of preschool-aged children
}

\begin{abstract}
Objectives: The aim of this study was to explore factors which mediated or moderated the effect of the Time2bHealthy online program for parents of preschool-aged children on body mass index (BMI) change. Study design: Mediation and moderation analyses of data from a two-arm parallel randomised controlled trial. Methods: Randomisation was conducted after baseline measures. The intervention group received an 11-week online program, and the comparison group received emailed links to information from an evidence-based parenting website. Data on the primary outcome (child BMI), potential mediators (energy intake, fruit and vegetable intake, discretionary food intake, physical activity, screen-time, sleep, child feeding, parent self-efficacy or parent role-modelling) and potential moderators (child age, parent age, parent income, parent education or parent living situation) were collected at baseline, 3 months and 6 months. PROCESS macro for SPSS was used to analyse possible mediators and moderators on BMI outcomes. Results: Despite significant food-related outcomes in the main analysis of this trial, no significant mediating or moderating effects were found for any hypothesised mediators or moderators. Conclusions: This study's null results could be explained by the high proportion of children in the healthy weight range, the study period not being long enough to detect change, the multicomponent nature of the intervention or the relatively small number of outcomes measured. Future childhood obesity interventions should continue to explore the effects of mediators and moderators on BMI and consider collecting data on a wide range of mediating and moderating factors to allow for comparison between studies to develop a better understanding of the factors contributing to successful interventions.

Disciplines

Education | Social and Behavioral Sciences

Publication Details

Hammersley, M. L., Okely, A. D., Batterham, M. J. \& Jones, R. A. (2019). Investigating the mediators and moderators of child body mass index change in the Time2bHealthy childhood obesity prevention program for parents of preschool-aged children. Public Health, 173 50-57.
\end{abstract}

This journal article is available at Research Online: https://ro.uow.edu.au/sspapers/4474 


\section{ABSTRACT}

Objectives: The aim of this study was to explore factors which mediated or moderated the effect of the Time2bHealthy online program for parents of preschool-aged children on BMI change.

Study design: Mediation and moderation analyses of data from a 2-arm parallel randomised controlled trial.

Methods: Randomisation was conducted post baseline measures. The intervention group received an 11-week online program and the comparison group received emailed links to information from an evidence-based parenting website. Data on the primary outcome (child BMI), potential mediators (energy intake, fruit and vegetable intake, discretionary food intake, physical activity, screen-time, sleep, child feeding, parent self-efficacy or parent rolemodelling) and potential moderators (child age, parent age, parent income, parent education or parent living situation) were collected at baseline, 3- and 6-months. PROCESS macro for SPSS was used to analyse possible mediators and moderators on BMI outcomes.

Results: Despite significant food-related outcomes in the main analysis of this trial, no significant mediating or moderating effects were found for any hypothesised mediators or moderators.

Conclusions: This study's null results could be explained by the high proportion of children in the healthy weight range, the study period not being long enough to detect change, the multi-component nature of the intervention or the relatively small number of outcomes measured. Future childhood obesity interventions should continue to explore the effects of mediators and moderators on BMI and consider collecting data on a wide range of mediating and moderating factors to allow for comparison between studies to develop a better understanding of the factors contributing to successful interventions. 
Keywords: Mediators, moderators, childhood obesity, BMI, eHealth

\section{INTRODUCTION}

Childhood obesity is a major problem worldwide. Childhood obesity tracks from early childhood to adulthood and can result in life-long health issues ${ }^{1}$ and well as significant indirect costs. ${ }^{2}$ Interventions which aim to treat and/or prevent childhood obesity have been implemented in a range of settings and modes of delivery, some demonstrating success in reducing obesity and/or obesity-related factors, while others have not. ${ }^{1,3}$ Although there has recently been a greater focus on investigating the specific mechanisms by which such interventions achieve outcomes, there is still limited research in this area, and it has been recommended that interventions include analyses of mediators and moderators. ${ }^{4,5}$ This is particularly important in the early childhood stage where behaviour change is paramount. Studies which explore the mediators and moderators of change are imperative as they assist in gaining a greater understanding of the contributing factors which may have an influence on an intervention achieving desired outcomes. Gaining insight into mediators and moderators that facilitate change can inform the design of more effective interventions, which can be more appropriately targeted.

There have been limited childhood obesity interventions which have explored mediators and moderators of body mass index (BMI), zBMI, weight or adiposity change. The few studies conducted in this area have identified mediators such as dietary intake ${ }^{6}$ and physical activityrelated factors, ${ }^{7}$ self-regulation, mood and self-efficacy. ${ }^{8}$ Moderators identified include age, ${ }^{9}$ baseline weight, ${ }^{10}$ baseline dietary intake factors, ${ }^{11}$ social and psychological problems, ${ }^{9,} 12$ and the built environment. ${ }^{13}$ Only one study has explored mediators of BMI change in an internet-based childhood obesity intervention, which found that parental life and family satisfaction mediated weight loss ${ }^{14}$ and only one study has explored both mediators and 
moderators of BMI change in childhood obesity interventions targeting preschool-aged children.

This paper explores potential mediators and moderators of BMI change in the Time2bHealthy study. The Time2bHealthy study was a randomised controlled trial (RCT) of a healthy lifestyle program for parents of 2-5 year old children conducted between 2016 and 2017. The main outcomes have been reported previously ${ }^{15}$. Briefly, there was no significant difference in child BMI (the primary outcome) between groups, but there was a significant reduction in BMI within the intervention group. A significant reduction in the frequency of discretionary food intake in the children in the intervention group compared to those in the comparison group was also reported. There was also a greater improvement in 'pressure to eat' child feeding practices and parent nutrition self-efficacy in the intervention group compared to the comparison group. The aim of the current paper was to further explore the data from the Time2bHealthy RCT to investigate if change in child BMI was mediated by changes in obesity-related variables or moderated by demographic characteristics. The hypothesis was that child BMI change at 6-months post-baseline would be mediated by change in obesityrelated variables (energy intake, fruit and vegetable intake, discretionary food intake, physical activity, screen-time, sleep, child feeding, parent self-efficacy or parent role-modelling) and/or moderated by demographic characteristics (child age, parent age, parent income, parent education or parent living situation). ).

\section{METHODS}

\section{Study Design}

The Time2bHealthy study was a two-arm parallel randomised controlled trial approved by the University of Wollongong Human Research Ethics Committee (HE15/354) and registered with the Australian and New Zealand Clinical Trials registry (ACTRN:12616000119493) 
http://www.anzctr.org.au/. All parent participants gave informed written consent. The trial was conducted between January 2016 and December 2017.

\section{Participant recruitment and eligibility}

Eligible individuals were 2-5-year-old children who were above the WHO 50th percentile for BMI for their age and sex and their parents. Children were excluded if they were taking medications or had a medical condition that could affect weight or restrict age-appropriate play. Parents were also required to have an existing Facebook account or agree to create one. Provisional eligibility was determined over the phone or via email and eligibility was confirmed at the face-to-face baseline appointment where height and weight were measured. Participants were recruited to the study from the Illawarra and surrounding areas in New South Wales and Melbourne, Victoria, Australia. Further details regarding the methods employed for this study have been previously published. ${ }^{15}$

\section{Randomisation}

Following baseline collection, participants were randomised into the intervention or comparison group. A computerised random number generator was used by a data manager who was not otherwise involved in the study to conduct the randomisation. The only individual who was informed of group allocation was the researcher responsible for implementing the intervention. Height and weight measurements were collected by trained and blinded data collectors at the follow-up data collection time-points.

\section{Time2bHealthy Intervention}

Details of the intervention have previously been published. ${ }^{15}$ In brief, the Time2bHealthy group received an 11-week online healthy lifestyle program, underpinned by Social Cognitive Theory, ${ }^{16}$ The intervention was designed using a backwards intervention mapping process to align the activities to the theory and target behaviours. ${ }^{17,18}$ Time2bHealthy comprised six 
modules (introduction, healthy eating (x2), physical activity, screen-time and sleep), followed by a 3-month maintenance period. The modules required participants to read content, watch videos, complete activities and set goals. A dietitian then provided feedback on the goals set. During the maintenance period, participants received fortnightly emails which revised the key information from each of the modules. Participants also had access to a closed (secret) Facebook group.

\section{Comparison Condition}

The comparison group received $11 \mathrm{x}$ weekly emails with links to information on similar topics on the evidence-based Raising Children Network website. Similar to the intervention group, during the maintenance period they received fortnightly emails which revised the information sent in the previous emails.

\section{Measures}

Data collection was via face-to-face visits with the child/parent dyads conducted at baseline, 3-months and 6-months. Child BMI was calculated using a standardised method. ${ }^{19}$ Height was measured to the nearest $0.1 \mathrm{~cm}$ using a stadiometer and weight was measured to the nearest $0.1 \mathrm{~kg}$ using a SECA scale. Height and weight measurements were then repeated and the mean taken to calculate BMI. Where height measurements differed by more than $0.5 \mathrm{~cm}$ and/or weight measurements differed by more than $0.5 \mathrm{~kg}$, a third measurement was taken.

Obesity-related behaviour and parent self-efficacy measures were collected from parents via an iPad including parent questionnaires on demographics (baseline only), food intake, screentime, sleep, self-efficacy, child feeding and role modelling. Questionnaires which had been assessed for validity and reliability were used where possible.

The food questionnaire (modified from the Eating and Physical Activity Questionnaire (EPAQ), which has been validated in the preschool age group ${ }^{20}$ ) assessed intake of fruit, 
vegetables and discretionary foods. Daily fruit and vegetable intake was measured on a continuous scale. A set of questions assessed frequency of intake of discretionary foods on an ordinal rating scale from never to 2 or more times per day (Cronbach’s alpha=0.68). Responses to questions on frequency of intake of takeaway or fast food; sugary cereals; potato chips or other salty snacks; sweets; cakes, doughnuts, sweet biscuits or muffins; and sugary drinks were then used to calculate a discretionary food score. A parent-reported 24-h recall of child dietary intake was conducted (using the ‘Easy Diet Diary’ app (Xyris Software (Australia) Pty Ltd)). Data from the 24-h recall was used to calculate kJ per kg of body weight.

Actigraph GT3X+ accelerometers (ActiGraph Corporation, Pensacola, FL) were used to measure physical activity. Monitors were worn by children for 7-days with an elasticised belt around their waist. Data were analysed in ActiLife version 6 (ActiGraph Corporation, Pensacola, FL). Accelerometers have been previously used in many physical activity studies in children and have been validated for use in preschool-aged children. ${ }^{21}$ Categorization of physical activity was conducted using the following cut-points; sedentary $<100$ counts/min, low light-intensity physical activity 101-800 counts/min, high light-intensity physical activity 801-1679 counts/min, moderate-intensity physical activity 1680-3367 count/min and vigorous-intensity physical activity $\geq 3368$ count/min. ${ }^{22,23}$

Screen-time was measured using a set of questions (based on previously used questions which have been assessed for reliability) ${ }^{24,25}$ to ascertain the usual number of hours of screen-time per day on weekends and weekdays, which were then used to calculate overall average time per day. Sleep duration was assessed via accelerometer and parent-reported questionnaire ${ }^{26}$ as usual hours per night. Parent-reported sleep duration was used for the purpose of these analyses due to poor compliance with night-time accelerometry. The questions used were modified from a tool which has been previously used in the preschool 
age group. ${ }^{26}$ Child feeding was assessed using the validated 'pressure to eat' and 'restriction' sub-scales from the validated Child Feeding Questionnaire ${ }^{27}$ and parent role modelling (developed after reviewing other questionnaires as there were no appropriate existing tools ${ }^{28 \text {, }}$ ${ }^{29}$ Cronbach's alpha=0.63)) was assessed using a set of questions on a five-point scale. Parent self-efficacy was scored using a set of questions on a 0 to 10 scale which were modified from a previously validated questionnaire ${ }^{30}$ (Cronbach’s alpha=0.89), Self-efficacy is a key component of Social Cognitive Theory. Parent self-efficacy has been found to be important in facilitating change in obesity-related behaviours in young children ${ }^{31}$ such as increasing fruit and vegetable intake, ${ }^{32-34}$ increasing physical activity, ${ }^{34}$ and limiting consumption of unhealthy foods. ${ }^{31,32,34,35}$

\section{Statistical analysis}

Power calculations for the Time2bHealthy study have been published previously. ${ }^{15}$ Mediation and moderation complete case analyses were conducted in IBM SPSS Statistics for Windows, version 25 (IBM Corp., Armonk, N.Y., USA) to explore whether child BMI change at 6 months post-baseline was mediated by changes in obesity-related variables at 3 months postbaseline or moderated by participant demographics at baseline. Baseline values and child age were included as covariates in the models.

Obesity-related variables hypothesised to mediate the effect of BMI change were included in a mediation model a priori. Change in fruit and vegetable intake, energy intake ( $\mathrm{kJ} / \mathrm{kg}$ body weight), discretionary food intake, high light, moderate and vigorous-intensity physical activity (LMVPA), screen-time, sleep duration, child feeding (restriction and pressure to eat sub-scales), parent self-efficacy and parent role modelling from baseline to 3-months were hypothesised to mediate change in BMI at 6 months (Figure 1). For the purpose of this analysis, ordinal data was treated as continuous. Model four of the PROCESS Macro for 
SPSS version 3.0 was used to calculate the pathways. Mediation procedures outlined by Hayes ${ }^{36}$ were used to guide the analysis. The direct effect of the intervention on change in obesity-related variables at 3-months was determined in pathway a. The association between change in the obesity-related variables at 3 months and BMI change at 6 months was determined in pathway b. The direct effect of the intervention on BMI change was determined in pathway c’. The indirect intervention effects were determined via pathway ab. Bootstrapped 95\% CIs were calculated to test if the indirect effect was significant. Mediation was determined to be significant if the CIs did not include zero.

Moderation analyses were conducted using model one of the PROCESS Macro for SPSS version 3.0. Moderation procedures outlined by Hayes ${ }^{36}$ were used to guide the analyses. Single moderation models were used for the baseline demographic variables of parent age, child age, parent income, parent education and parent living situation (with/without partner) to determine if there was a moderating effect of any of these variables on BMI change at 6 months.

\section{RESULTS}

Initial contact was received from 372 parents who enquired about the study and received the information sheet. 159 participants stated that they were interested and after screening 104 parent/child dyads were potentially eligible and invited to attend an initial appointment. Ninety-three parent/child dyads attended this appointment and 86 were confirmed to be eligible. Forty-two dyads were randomised to the intervention group and 44 to the comparison group. Most participants were in the healthy weight range (91\%), 63\% had a university degree and $85 \%$ were married or with partner. The study had a retention rate of 91\%, with one participant withdrawing and 7 lost to follow-up. Further information regarding 
baseline demographics and participant flow have been previously reported by [REMOVED FOR BLIND PEER REVIEW]. ${ }^{15}$

\section{Mediation and Moderation analyses}

The results of the mediation analyses are displayed in Table $\mathbf{1}$ and the results of the moderation analysis are shown in Table 2. Despite significant results previously reported in the main outcome analyses for frequency of discretionary food intake, child feeding pressure to eat and parent nutrition self-efficacy, none of the hypothesised obesity-related variables were significant mediators of BMI change at 6-months. Furthermore, none of the hypothesised participant characteristic variables were significant moderators of BMI change at 6-months.

\section{DISCUSSION}

This current paper exploring the mediators and moderators of BMI change at 6 months postbaseline found no significant effect of the hypothesised mediators and moderators of the intervention on BMI change. There is an identified need for interventions that explore the mediators and moderators of change in childhood obesity interventions ${ }^{4,5}$ and to date there have been few studies examining mediating and moderating effects of an intervention on BMI change. ${ }^{6-14}$ There is a particular need for these analyses in childhood obesity studies using eHealth (technology supported healthcare and health interventions) and those involving preschool-aged children., ${ }^{7,14}$ As eHealth is a rapidly growing area of research, the need to investigate the mechanisms by which these interventions work and who they work for is particularly important.

The aim of this paper was to explore mediators and moderators of child BMI change at 6 months post-intervention in the Time2bHealthy RCT. The main outcomes of the RCT indicated that although there was no significant difference between groups in BMI change, 
there was a significant improvement in BMI within the intervention group.${ }^{15}$ While the original study found no significant difference between groups in BMI change, it is still worthwhile exploring potential moderators and mediators. The effect of an intervention on a mediator variable can be greater than the direct effect on the outcome variable and therefore may be a stronger indirect effect of the intervention. ${ }^{6}$ Mediation results may also indicate the future potential of the intervention to effect the main outcome. ${ }^{37}$ Exploring potential moderators in interventions where there is no significant effect of the intervention on the main outcome is useful for uncovering opposing effects of an intervention based on moderating effects of participant characteristics which would not be apparent otherwise. ${ }^{37}$ As the majority of children in the study were in the healthy weight range, there may have been a dilution effect on BMI, which could have impacted on both the null findings in the main outcomes analysis and the null findings in the mediation and moderation analysis. Furthermore, due to the breadth of content of the Time2bHealthy intervention and because there were a limited number of mediators and moderators for which we collected data and included in these analyses, it is possible that other mediators and moderators that we have not assessed were significant in facilitating BMI change. In the only other childhood obesity eHealth intervention which has explored mediators, White et $\mathrm{al}^{14}$ found that parent life and family satisfaction were significant mediators of weight loss in a family-focussed eHealth childhood obesity intervention for 11- to 15-year-old children involving nutrition education and behaviour change strategies for adolescents and their parents.

. The results of the only two traditionally delivered (i.e. face-to-face) studies have been mixed, ${ }^{7,38}$ with only one study reporting a significant mediation and moderation result. zBMI change was moderated by socioeconomic status and targeted sedentary behaviour had a significant mediating effect on zBMI in a study which aimed to reduce television viewing and computer use. ${ }^{7}$ The intervention targeted sedentary behaviour only and was therefore quite 
different to the multi-behaviour intervention design employed in the Time2bHealthy study, which also focussed on healthy eating, physical activity and sleep. Unlike Time2bHealthy, the study also enforced mandatory sedentary behaviours limits (i.e., capped time spent in sedentary behaviours), provided children with financial incentives for reducing TV and computer use and fitted a device to TVs and computer monitors preventing their use after the screen-time 'budget' was exhausted. The study was also conducted over a 2-year period and therefore much longer than the Time2bHealthy intervention. It is possible that more time would be required to demonstrate change in BMI, particularly given the age of the children, and had a longer follow-up period been included in the Time2bHealthy study, perhaps mediators of change would have been detected.

A number of mediators and moderators have been found to have an effect in previous studies of older children which were not included in the mediation and moderation models in our analyses. Factors such as self-regulation, mood, child self-efficacy, ${ }^{8}$ aerobic fitness, ${ }^{39}$ resistance training self-efficacy, physical activity behavioural change ${ }^{10}$ and family factors ${ }^{14}$ have mediated BMI effects. Characteristics such as social adjustment/problems, ${ }^{9,}{ }^{12}$ anxiety, ${ }^{9}$ built environment factors, ${ }^{13}$ baseline energy dense food intake, parent concern over own weight, and parent child acceptance ${ }^{11}$ have moderated the effect on BMI outcomes. While previous studies have had specific areas of focus, due to the wide range of mediators and moderators explored in studies, it is difficult to make comparisons and it is therefore recommended that future interventions investigate a broader range of mediators and moderators to enable results to be compared between studies. The mixed results of the mediating and moderating factors of childhood obesity interventions on BMI outcomes in the current literature demonstrate that more studies which incorporate mediation and moderation analyses are needed, particularly in interventions targeting younger children and eHealthbased studies. 


\section{Strengths and Limitations}

This is one of the first papers to explore mediators and/or moderators of BMI change in a childhood obesity intervention in the preschool age group and the first to explore both mediators and moderators of BMI change in an eHealth childhood obesity study in any age group. There are some limitations of this study. As mentioned, the majority of children (91\%) in the study were in the healthy weight range and there was a possible dilution effect on BMI. Therefore, it could be argued that the eligibility criteria may have been too broad. However, targeting children who are at may be risk of becoming weight is important in long-term obesity prevention. Also, had the eligibility criteria been restricted to children with overweight and obesity, the trial may not have been able to proceed if a minimum sample size had not been achieved. The sample was not perfectly representative of the general Australian population as it contained a higher proportion of participants in the healthy weight range compared to the overall Australian population (where approximately $80 \%$ of children aged 2-4 years are in the healthy weight range). ${ }^{40}$ Participants were also generally more educated (63\% possessing a university degree compared to 23\% of the general Australian population $)^{41}$ and more likely to be married/with partner (85\%) than Australian families with children in general $(74 \%)^{42}$. Heterogeneity was not assessed in this study. It is possible that both response to the intervention and the mediators and moderators of change varied between participants due to individual characteristics, an avenue that could be explored in future studies. The sample size was smaller than planned, despite strategies implemented to enhance recruitment as previously described ${ }^{15}$, which therefore would have affected the statistical power. This mediation and moderation analysis was a complete case analysis. The main outcomes analysis for this study ${ }^{15}$ used an intention-to-treat design and a complete case analysis was also undertaken. Few differences were found between these two analyses of the main outcomes and we therefore propose that had an intention-to-treat design been used for 
this and moderation analysis, similar results would have been found. It is possible that questionnaire-based data could be intentionally or unintentionally misreported by parents, a common issue to many other studies. ${ }^{43,44}$ Finally, the number of mediators and moderators tested were limited by the data that were collected in the main study.

In conclusion, this exploratory analysis of the mediators and moderators of the Time2bHealthy childhood obesity intervention on BMI showed null results. There is a lack of studies in this area, particularly in younger children and in the field of eHealth. Further research is required, exploring a wider range of factors to gain greater insight into the mechanisms by which interventions achieve or don't achieve outcomes, which can be used to better inform policy and the design of more successful interventions.

\section{STATEMENT OF ETHICS APPROVAL}

The Time2bHealthy RCT was approved by the University of Wollongong Human Research Ethics Committee (HE15/354).

\section{FUNDING}

This research has been conducted with the support of the Australian Government Research Training Program Scholarship. The work was also supported by funding from the Telstra Foundation and Australian Health Management. The funding body was not involved in the design, data collection, analysis, interpretation or writing.

\section{COMPETING INTERESTS}

None.

\section{CONTRIBUTORS}

MLH contributed to designing the research, conducted the research, analysed data and wrote the paper. RAJ contributed to designing the research, conducted the follow-up data collection 
and edited the manuscript. MJB contributed to designing the research, provided input and advice on the statistical analysis plan, data interpretation and edited the manuscript. ADO contributed to designing the research and edited the manuscript. All authors read and approved the final manuscript. 


\section{REFERENCES}

1. Luttikhuis HO, Baur L, Jansen H, Shrewbury VA, O’Malley C, Stolk RP, Summerbell CD. Interventions for treating obesity in children. Cochrane Database Syst Rev 2009. 1. https://doi.org/ 10.1002/14651858.CD001872.pub2.

2. Bockerman P, Cawley J, Viinikainen J, Lehtimaki T, Rovio S, Seppala, Pehkonen J, Raitakari O. The effect of weight on labor marekt outcomes: an application of genetic instrumental variables. Health Econ 2019;28:65-77

3. Waters E, De Silva-Sanigorski AM, Hall BJ, Brown T, Campbell KJ, Gao Y, Armstrong R, Prossor L, Summerbell CD. Interventions for preventing obesity in children. Cochrane Database Syst Rev 2011. https://doi.org/10.1002/14651858.CD001871.pub3.

4. Wilfley DE, Tibbs TL, van Buren DJ, Reach KP, Walker MS, Epstein LH. Lifestyle interventions in the treatment of childhood overweight: a meta-analytic review of randomized controlled trials. Health Psychol 2007;26:521-532. https://doi.org/10.1037/0278-6133.26.5.521

5. Whittemore R., Chao A, Popick R, Grey M. School-based internet obesity prevention programs for adolescents: a systematic literature review. Yale J Biol Med 2013;86:4962.

6. Yildirim M., Singh AS, te Velde SJ, van Stralen MM, MacKinnon DP, Brug J, van Mechelen W, Chinapaw MJ. Mediators of longitudinal changes in measures of adiposity in teenagers using parallel process latent growth modeling. Obesity (Silver Spring) 2013:21:2387-95. https://doi.org/10.1002/oby.20463.

7. Epstein LH, Roemmich JN, Robinson JL, Paluch RA, Winiewicz, DD, Fuerch JH, Robinson TN. A randomized trial of the effects of reducing television viewing and 
computer use on body mass index in young children. Arch Pediatr Adolesc Med 2008;162:239-245. https://doi.org/10.1001/archpediatrics.2007.45.

8. Annesi JJ, Walsh SM, Greenwood BL, Mareno N, Unruh-Rewkowski JL. Effects of the Youth Fit 4 Life physical activity/nutrition protocol on body mass index, fitness and targeted social cognitive theory variables in 9- to 12-year-olds during after-school care. J Paediatr Child Health 2017;53:365-373. https://doi.org/10.1111/jpc.13447.

9. Burke NL, Shomaker LB, Brady S, Reynolds JC, Young JF, Wilfley DE, Sbrocco T, Stephens M, Olsen CH, Yanovski JA, Tanofsky-Kraff M. Impact of age and race on outcomes of a program to prevent excess weight gain and disordered eating in adolescent girls. Nutrients 2017;9(9):947. https://doi.org/10.3390/nu9090947.

10. Lubans DR., Morgan PJ, and Callister R, Potential moderators and mediators of intervention effects in an obesity prevention program for adolescent boys from disadvantaged schools. J Sci Med Sport 2012;15:519-525. https://doi.org/10.1016/j.jsams.2012.03.011.

11. Epstein LH, Paluch RA, Beecher MD, Roemmich JN. Increasing healthy eating vs. reducing high energy-dense foods to treat pediatric obesity. Obesity (Silver Spring 2008;16:318-326. https://doi.org/10.1038/oby.2007.61.

12. Wilfley DE, Stein RI, Saelens BE. Efficacy of maintenance treatment approaches for childhood overweight: a randomized controlled trial. JAMA 2007;298:1661-1673.

13. Epstein LH, Raja S, Daniel TO, Paluch RA, Wilfley DE, Saelens BE, Roemmich JN. The built environment moderates effects of family-based childhood obesity treatment over 2 years. Ann Behav Med 2012;44:248-58. https://doi.org/10.1001/jama.298.14.1661.

14. White MA, Martin PD, Newton RL, Walden HM, York-Crowe EE, Gordon ST, Ryan DH, Williamson DA. Mediators of weight loss in a family-based intervention 
presented over the internet. Obes Res 2004;12:1050-9. https://doi.org/10.1038/oby.2004.132.

15. Hammersley ML, Jones RA, Okely AD (2017). Time2bHealthy - An online childhood obesity prevention program for preschool-aged children: A randomised controlled trial protocol. Contemporary Clinical Trials. 61:73-80. doi: 10.1016/j.cct.2017.07.022

16. Bandura, A., Social Cognitive Theory, in R. Vasta (ed) Annals of child development Volume 6. Six Theories of Child Development. Greenwich:JAI Press. 1989;1-60.

17. Cornelius M, Armel KC, Hoffman K, Allen L, Bryson SW, Desai M, Robinson TN. Increasing energy- and greenhouse gas-saving behaviors among adolescents: a school-based cluster-randomized controlled trial. Energ Effic 2014, 7:217-242. doi: 10.1007/s12053-013-9219-5

18. Robinson TN, Borzekowski DLG. Effects of the SMART classroom curriculum to reduce child and family screen time. J Commun 2006, 56:1-26. doi: 10.1111/j.14602466.2006.00001.x

19. National Health and Medical Research Council. Clinical Practice Guidelines for the Management of Overweight and Obesity in Adults, Adolescents and Children. Melbourne, Australia: National Health and Medical Research Council. 2013:

20. Bennett CA, de Silva-Sanigorski AM, Nichols M, Bell AC, Swinburn BA. Assessing the intake of obesity-related foods and beverages in young children: comparison of a simple population survey with 24 hr-recall. Int J Behav Nutr Phys Act 2009;6:71. https://doi.org/10.1186/1479-5868-6-71.

21. De Vries SI, Van Hirtum HW, Bakker I, Hopman-Rock M, Hirasing RA, Van Mechelen W. Validity and reproducibility of motion sensors in youth: a systematic update. Med Sci Sports 2009, 41:818-827. doi: 10.1249/MSS.0b013e31818e5819 
22. Pate RR, Almeida MJ, McIver KL, Pfeiffer KA, Dowda M. Validation and calibration of an accelerometer in preschool children. Obesity (Silver Spring) 2006;14:2000-6. https://doi.org/10.1038/oby.2006.234.

23. Carson V, Ridgers ND, Howard BJ, Wilkler EAH, Healy GN, Owen N, Dunstan DW, Salmon J. Light-intensity physical activity and cardiometabolic biomarkers in us adolescents. PLOS One 2013;8:e71417. https://doi.org/10.1371/journal.pone.0071417.

24. Downing KL, Hinkley T, Hesketh KD. Associations of parental rules and socioeconomic position with preschool children's sedentary behaviour and screen time. J Phys Act Health 2015;12:515-21. https://doi.org/10.1123/jpah.2013-0427.

25. Hinkley T, Salmon J, Okely A, Crawford D, Hesketh K. The HAPPY study: development and reliability of a parent survey to assess correlates of preschool children's physical activity. J Sci Med Sport 2012;15:407-17. https://doi.org/10.1016/j.jsams.2011.12.009.

26. Sneddon P, Peacock GG, Crowley SL. Assessment of sleep problems in preschool aged children: an adaptation of the children's sleep habits questionnaire. Behav Sleep Med 2013;11:283-96. https://doi.org/10.1016/10.1080/15402002.2012.707158.

27. Birch LL, Fisher JO, Grimm-Thomas K, Markey CN, Sawyer R, Johnson SL. Confirmatory factor analysis of the Child Feeding Questionnaire: a measure of parental attitudes, beliefs and practices about child feeding and obesity proneness. Appetite 2001;36:201-10. https://doi.org/10.1006/appe.2001.0398.

28. Palfreyman Z, .Haycraft E, Meyer C. Development of the Parental Modelling of Eating Behaviours Scale (PARM): links with food intake among children and their mothers. Matern Child Nutr 2014;10;617-29. https://doi.org/10.1111/j.17408709.2012.00438.x. 
29. Gattshall ML, Shoup JA, Marshall JA, Crane LA, Estabrooks PA. Validation of a survey instrument to assess home environments for physical activity and healthy eating in overweight children. Int J Behav Nutr Phys Act 2008;5:3. https://doi.org/10.1186/1479-5868-5-3.

30. Bohman B, Ghaderi A, Rasmussen F. Psychometric properties of a new measure of parental self-efficacy for promoting healthy physical activity and dietary behaviors in children. Eur J Psychol Assess 2013;29:291-298. https://doi.org/10.1027/10155759/a000159.31.

31. Bohman B, Rasmussen F, Ghaderi A. Development and psychometric evaluation of a context-based parental self-efficacy instrument for healthy dietary and physical activity behaviors in preschool children. Int J Behav Nutr and Phys Act 2016, 13:110. doi: 10.1186/s12966-016-0438-y

32. Campbell K, Hesketh K, Silverii A, Abbott G. Maternal self-efficacy regarding children's eating and sedentary behaviours in the early years: associations with children's food intake and sedentary behaviours. Int J Pediatr Obes 2010, 5:501-508. doi: $10.3109 / 17477161003777425$

33. Koh, G. A., Scott, J. A., Woodman, R. J., Kim, S. W., Daniels, L. A., \& Magarey, A. M. Maternal feeding self-efficacy and fruit and vegetable intakes in infants. Results from the SAIDI study. Appetite 2014, 81:44-51. doi: 10.1016/j.appet.2014.06.008

34. Rohde JF, Bohman B, Berglind D, Hansson LM, Frederiksen P, Mortensen EL, et al. Cross-sectional associations between maternal self-efficacy and dietary intake and physical activity in four-year-old children of first-time Swedish mothers. Appetite 2018, 125:131-138. doi: 10.1016/j.appet.2018.01.026 
35. Jago, R., Sebire, S. J., Edwards, M. J., \& Thompson, J. L. (2013). Parental TV viewing, parental self-efficacy, media equipment and TV viewing among preschool children. Eur J Pediatr 2013, 172: 1543-1545. doi: 10.1007/s00431-013-2077-5

36. Hayes AF. Introduction to Mediation, moderation, and conditional process analysis: a regression-based approach. 2nd Ed ed. New York: Guildford Press. 2017.

37. MacKinnon DP. Integrating mediators and moderators in research design. Res Soc Work Pract. 2011;21:675-681. https://doi.org/10.1177/1049731511414148.

38. Enö Persson J, Bohman B, Tynelius P, Rasmussen F, Ghaderi, A. Prevention of childhood obesity in child health services: follow-up of the PRIMROSE trial. Child Obes 2017;14:99-105. https://doi.org/10.1089/chi.2017.0117.

39. Maddison R., Mhurchu CN, Jull A, Prapavessis H, Foley LS, Jiang Y. Active video games: the mediating effect of aerobic fitness on body composition. Int J Behav Nutr Phys Act 2012;9:54. https://doi.org/10.1186/1479-5868-9-54.

40. Australian Institute of Health and Welfare. A picture of overweight and obesity in Australia 2017. Cat. no.PHE 216. Canberra, Australia: AIHW; 2017

41. Australian Bureau of Statistics. Australians pursuing higher education in record numbers, http://www.abs.gov.au/AUSSTATS/abs@.nsf/mediareleasesbytitle/1533FE5A8541D 66CCA2581BF00362D1D?OpenDocument; 2017 [accessed 26 February 2019]

42. Australian Bureau of Statistics. 2016 Census quickstats, http:/quickstats.censusdata.abs.gov.au/census_services/getproduct/census/2016/quick stat/036; 2017 [accessed 26 February 2019].

43. Gemming L, Jiang Y, Swinburn B, Utter J, Mhurchu CN. Under-reporting remains a key limitation of self-reported dietary intake: an analysis of the 2008/09 New Zealand Adult Nutrition Survey. Eur J Clin Nutr 2014;68:259-64. https://doi.org/10.1038/ejcn.2013.242. 
44. Poslusna K, Ruprich J, de Vries JH, Jakubikova M, van’t Veer. Misreporting of energy and micronutrient intake estimated by food records and 24 hour recalls, control and adjustment methods in practice. Br J Nutr 2009;101 (Suppl 2):S73-85. https://doi.org/0.1017/S0007114509990602. 